\title{
dspace.vutbr.cz
}

\section{Adjustable Multiphase Sinusoidal Oscillator with Fractional-Order Elements}

DVOŘÁK, J.; KUBÁNEK, D.; KOTON, J.; JEŘÁBEK, J.; SMÉKAL, D.

Proceedings of the 10th IEEE International Congress on Ultra Modern Telecommunications and Control Systems (ICUMT 2019), pp. 1-6

elSBN: 978-1-7281-5764-1

ISSN: 2157-023X

DOl: https://doi.org/10.1109/ICUMT48472.2019.8970842

Accepted manuscript

(C)2019 IEEE. Personal use of this material is permitted. Permission from IEEE must be obtained for all other uses, in any current or future media, including reprinting/republishing this material for advertising or promotional purposes, creating new collective works, for resale or redistribution to servers or lists, or reuse of any copyrighted component of this work in other works. J. Dvorak, D. Kubanek, J. Koton, J. Jerabek and D. Smekal, " Adjustable Multiphase Sinusoidal Oscillator with Fractional-Order Elements ", Proceedings of the 10th IEEE International Congress on Ultra Modern Telecommunications and Control Systems (ICUMT 2019), pp. 1-6, 2019. DOI: $10.1109 /$ ICUMT48472.2019.8970842. Final version is available at https://ieeexplore.ieee.org/document/8970842 


\title{
Adjustable Multiphase Sinusoidal Oscillator with Fractional-Order Elements
}

\author{
Jan Dvorak, David Kubanek, Jaroslav Koton, Jan Jerabek, David Smekal \\ Department of Telecommunications \\ Brno University of Technology \\ Brno, Czech Republic \\ dvorakjan@feec.vutbr.cz
}

\begin{abstract}
This paper presents a fractional-order multiphase oscillator with adjustable FO (Frequency of Oscillation). The oscillator utilizes the following components: CCTA (Current Conveyor Transconductance Amplifier), OTA (Operational Transconductance Amplifier), auxiliary OTRA (Operational Transresistance Amplifier), two FOC (Fractional-Order Capacitors) and auxiliary resistors. The proposed structure provides three voltage outputs and their inverted variants for three selected values of $\alpha=$ $0.3,0.5$, and 0.8 of FOC. The FO is tuned by changing the values of the transconductances of the OTA and CCTA. The features of the presented circuit were verified by PSpice simulations with behavioural models of the active elements. Analysis of the dependence of the values of the transconductances on values of $\alpha$ and frequency is provided. A THD (Total Harmonic Distortion) and parasitic analysis of the circuit was also made.
\end{abstract}

Index Terms - fractional-order oscillator, fractional-order capacitor, THD, multiphase oscillator

\section{INTRODUCTION}

The fractional-order systems become popular topic of many scientific groups from many research sectors. These systems bring greater variability in the design of functional systems and in setting their parameters in comparison with conventional integer-order systems [1]. The fractional-order systems can be used in many diverse scientific fields. For example in cryptography for generating random numbers [2], in biology for modelling of biological phenomena [3], in agriculture for analysis of the fruits or vegetable properties [4], in electrical engineering for design of the precise controllers [5], frequency filters [6] and oscillators [7]-[11].

Fractional-order oscillators are characterized by arbitrary phase shift of output signals in multiples of $\alpha \pi / 2$, where the parameter $\alpha$ is non-zero positive number in range from $0<\alpha<1$ [8]. Note that standard oscillators provide phase shift of output signals only in multiples of $\pi / 2$. The design of the fractional-order oscillators is based on usage of the fractional-order passive element (FOE) in the circuit structure. Most often fractional-order capacitor (FOC) is used, where the slope of impedance attenuation of the FOC equals to

This Article is based upon work from COST Action CA15225, a network supported by COST (European Cooperation in Science and Technology). Research described in this paper was financed by Czech Science Foundation under grant no. 19-24585S, by the Inter-Cost Program under grant LTC18022 and National Sustainability Program under grant LO1401. For the research, infrastructure of the SIX Center was used.
$20 \alpha \mathrm{dB} /$ decade and phase shift is $-\alpha \pi / 2$ [15]. The impedance of the FOC is described by the following equation:

$$
Z_{\mathrm{C} \alpha}=\frac{1}{Y_{\mathrm{C} \alpha}}=\frac{1}{\boldsymbol{s}^{\alpha} C_{\alpha}}=\frac{1}{(j \omega)^{\alpha} C_{\alpha}},
$$

where the $C_{\alpha}$ is called as pseudocapacitance with unit $\mathrm{F} / \sec ^{1-\alpha}$ and $\boldsymbol{s}$ is Laplacian operator [1], [7]. The value of the pseudocapacitance is calculated by $C_{\alpha}=C_{\text {eq }} \omega^{1-\alpha}$, where $C_{\text {eq }}$ is value of the conventional capacitor and $\omega$ is angular frequency. The $j^{\alpha}$ can be substituted as follows:

$$
j^{\alpha}=\cos (\alpha \pi / 2)+\mathrm{j} \sin (\alpha \pi / 2) .
$$

The paper [8] presents fractional-order Colpitts oscillator based on CMOS transconductors providing two voltage outputs. The simple proposal of the fractional-order oscillator with two voltage outputs based on differential voltage current conveyor transconductance amplifier (DVCCTA) and variable gain amplifier (VGA) employing impedance converter is presented in paper [9]. The main disadvantage of the design of the circuit containing the FOEs is commercial unavailability of these passive elements. Only special prototypes are fabricated [11]. These prototypes were used in [10] and [11] to design the fractional-order oscillators. The fractional-order oscillator of orders 2.6 and 2.7 with one voltage output designed with operational amplifier AD797 is presented and verified by measurements in [10]. In paper [11], the fractional-order Hartley oscillator designed by operational amplifier AD817 is analysed and verified also by measurements.

The proposal of the fractional-order oscillator with adjustable FO (Frequency of Oscilation) is presented and analysed in this paper. The proposed oscillator provides three voltage outputs with phase shift equal to multiple of $\alpha \pi / 2$ and electronically controllable FO. The analysis of the values of transconductances versus values of $\alpha$ and FO is also presented in this paper. The advantage of our solution is minimal number of the passive parts and active elements, control of the FO without affecting the condition of oscillation and total of six voltage outputs. Also advanced parasitic analysis of the active elements is provided, which was not performed in any of the before mentioned papers. Any before mentioned paper does not provide deep parasitic analysis of the proposed fractionalorder oscillator. The FO of the presented circuit is tuned 
by changing values of the transconductances of the CCTA (Current Conveyor Transconductance Amplifier) and OTA (Operational Transconductance Amplifier). The fractionalorder oscillator was proposed by the FOCs used instead both of conventional capacitors in the structure. The function of the proposed oscillator was verified using PSpice simulations with non-ideal models of the used active elements.

\section{Proposal of FractionAL-ORdER OSCILlATOR}

The scheme of the proposed fractional-order oscillator providing three voltage outputs $v_{1}, v_{2}, v_{3}$ and their inverted variants $v_{1}^{\prime}, v_{2}^{\prime}, v_{3}^{\prime}$ is depicted in Fig. 1 . The circuit has been designed with three active elements: CCTA [12], OTA [13], and auxiliary OTRA (Operational Transresistance Amplifier) providing required transfers and loops [14]. The function of the CCTA is described by the following equations [12]: $i_{\mathrm{OUT}+}=$ $-i_{\mathrm{OUT}-}=g_{\mathrm{m}} v_{\mathrm{Z}+}, v_{\mathrm{X}}=v_{\mathrm{Y}}$ and $i_{\mathrm{Z}+}=-i_{\mathrm{Z}-}=i_{\mathrm{X}}$, where parameter $g_{\mathrm{m}}$ is transconductance. The OTA is described by relation: $i_{\mathrm{OUT}}=g_{\mathrm{m}}\left(v_{\mathrm{IN}+}-v_{\mathrm{IN}-}\right)$. The OTRA can be easily described as follows: $v_{\mathrm{Z}+}=-v_{\mathrm{Z}-}=R_{\mathrm{m}} i_{\mathrm{IN}}$.

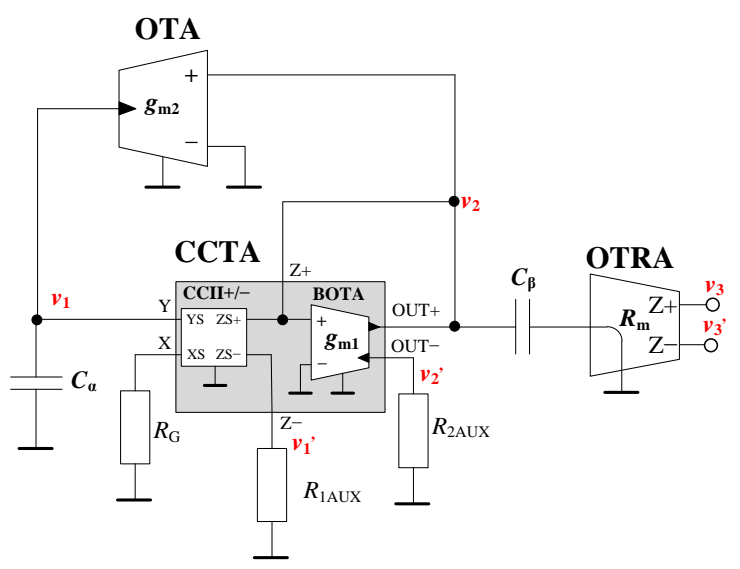

Fig. 1. Scheme of the proposed multiphase oscillator with six voltage outputs implemented with the CCTA, OTA and OTRA active elements.

The characteristic equation of the proposed fractional-order oscillator is given by:

$$
\boldsymbol{s}^{\alpha+\beta} C_{\alpha} C_{\beta}-\boldsymbol{s}^{\alpha} C_{\alpha} g_{\mathrm{m} 1}+\frac{g_{\mathrm{m} 2}}{R_{\mathrm{G}}}=0,
$$

where the substitution $s=j \omega$ change the characteristic equation as follows:

$$
j^{\alpha+\beta} \omega_{0}{ }^{\alpha+\beta} C_{\alpha} C_{\beta}-j^{\alpha} \omega_{0}{ }^{\alpha} C_{\alpha} g_{\mathrm{m} 1}+\frac{g_{\mathrm{m} 2}}{R_{\mathrm{G}}}=0,
$$

The real and imaginary parts of characteristic equation obtained from (4) are as follows:

$$
\begin{array}{r}
\Re: \omega_{0}{ }^{\alpha+\beta} C_{\alpha} C_{\beta} \cos \left[\frac{\pi}{2}(\alpha+\beta)\right]- \\
\omega_{0}{ }^{\alpha} C_{\alpha} g_{\mathrm{m} 1} \cos \left(\frac{\pi}{2} \alpha\right)+\frac{\mathrm{g}_{\mathrm{m} 2}}{\mathrm{R}_{\mathrm{G}}}=0, \\
\Im: \omega_{0}{ }^{\alpha+\beta} C_{\alpha} C_{\beta} \sin \left[\frac{\pi}{2}(\alpha+\beta)\right]- \\
\omega_{0}{ }^{\alpha} C_{\alpha} g_{\mathrm{m} 1} \sin \left(\frac{\pi}{2} \alpha\right)=0 .
\end{array}
$$

The relations for calculation of values of the transconductances $g_{\mathrm{m} 1}$ and $g_{\mathrm{m} 2}$ are obtained by rearranging the equations (5) and (6) to:

$$
\begin{gathered}
g_{\mathrm{m} 1}=\frac{\omega_{0}{ }^{\beta} C_{\beta} \sin \left[\frac{\pi}{2}(\alpha+\beta)\right]}{\sin \left(\frac{\pi}{2} \alpha\right)} \\
g_{\mathrm{m} 2}=\frac{\omega_{0}^{\alpha+\beta} C_{\alpha} C_{\beta} \sin \left(\frac{\pi}{2} \beta\right) \mathrm{R}_{\mathrm{G}}}{\sin \left(\frac{\pi}{2} \alpha\right)},
\end{gathered}
$$

The equations describing relationship between the voltages $v_{1}, v_{2}$, and $v_{3}$ of the circuit from Fig. 1 are the following:

$$
\begin{gathered}
v_{1}=\frac{v_{2} g_{\mathrm{m} 2}}{\omega_{0} \alpha C_{\alpha}} e^{-j \frac{\pi}{2} \alpha}, \\
v_{3}=v_{2} R_{\mathrm{m}} \omega_{0}^{\beta} C_{\beta} e^{j \frac{\pi}{2} \beta},
\end{gathered}
$$

where the phase shift between $v_{1}$ and $v_{2}$ is $-\alpha \pi / 2$, phase shift between $v_{3}$ and $v_{2}$ is $\beta \pi / 2$ and between $v_{1}$ and $v_{3}$ is $(\alpha+\beta) \pi / 2$.

For computing of the initial values of the oscillator parameters, the initial pole frequency and values of the $\alpha, \beta$ is important. In Fig. 2 (a) the Matlab simulations of dependence of values of the $g_{\mathrm{m} 1}$ on selected frequency and $\alpha=\beta$ (we consider $\alpha=\beta$ for simplification) is depicted. The analysis is performed based on equations (7) and (1) for values of the pole frequencies in range from 0 to $100 \mathrm{kHz}$, values of $\alpha=\beta$ in range from 0 to 1 and value of equivalent capacitance is $6.8 \mathrm{nF}$. In Fig. 2 (b) is shown the dependence of the values of $g_{\mathrm{m} 2}$ on frequency. Note that values of the $g_{\mathrm{m} 2}$ are dependent only on frequency as is obvious from substitution the equation $C_{\alpha}=C_{\text {eq }} \omega^{1-\alpha}$ to (8). These analysis are very helpful in case of selecting the initial values of the oscillator parameters. For example in selecting the initial pole frequency and values of the $\alpha, \beta$ of the proposed circuit.

The tuning of the FO can be performed by changing values of the $g_{\mathrm{m} 1}$ and $g_{\mathrm{m} 2}$ as is seen from following equation:

$$
\omega_{0}=\left[\frac{g_{\mathrm{m} 1}}{2 C_{\alpha} \cos \left(\frac{\pi}{2} \alpha\right)}\right]^{1 / \alpha}=\left[\frac{g_{\mathrm{m} 2}}{R_{\mathrm{G}} C_{\alpha}{ }^{2}}\right]^{1 / 2 \alpha} .
$$

Note that the condition of oscillation is fulfilled when the whole equation (11) is valid.

\section{Circuit Simulations}

The design correctness of the proposed fractional-order multiphase oscillator from Fig. 1 was verified by simulations in OrCAD PSpice with suitable behavioural models of the used active elements. The OTA and CCTA are in this paper implemented by UCCs (Universal Current Conveyors) with resistor $R$, representing the transconductance with value $g_{\mathrm{m}}=1 / R$, connected to $\mathrm{X}$ current terminal [13]. The simulation model 


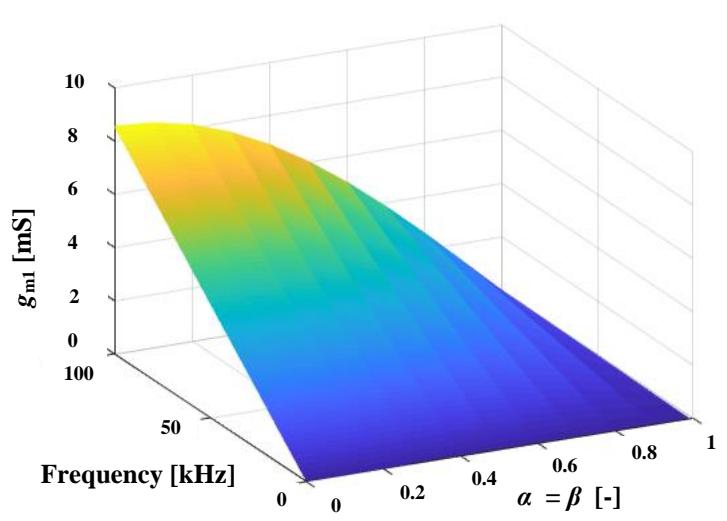

(a)

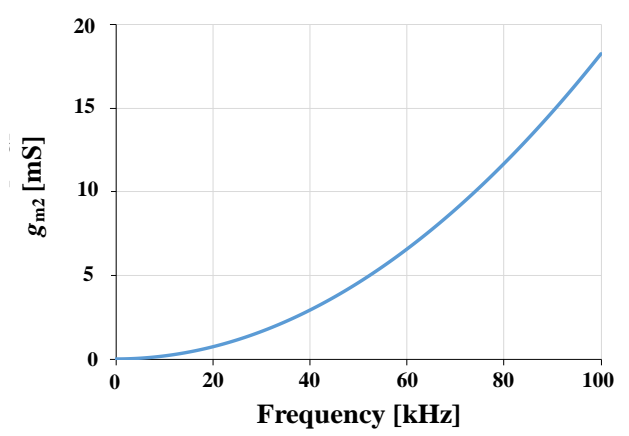

(b)

Fig. 2. Demonstration of the dependence of the values of transconductances on frequency and parameters $\alpha=\beta$ : (a) $g_{\mathrm{m} 1}$, (b) $g_{\mathrm{m} 2}$.

of the UCC is designed based on measurement of UCCN1B_0520 chips [16]. The OTRA is implemented by UCCs and $\mathrm{CCII}+/-$ with resistor $R_{\mathrm{m}}$ connected to the $\mathrm{X}$ terminal. The UCC chip havs been developed by Brno University of Technology and by ON Semiconductor Brno.

Both of capacitors are in the structure replaced by fractional-order capacitors with variable values of the order.

For fractional-order oscillator design presented in this paper, the FOC approximation through suitable and fully sufficient RC network [15] is used. The approximation of the FOE is dependent on selected type of the RC structure, number of the RC sections, type of the calculation method, value of the $\alpha$, and frequency band of the approximation validity [7], [8], [15]. The Foster I, Foster II, Cauer I, and Cauer II are types of RC structures most often used to approximate the FOC. In case of calculation of the values of passive parts in the selected RC structure, the Oustaloup's or CFE (Continuous Fraction Expansion) methods are most commonly used [7], [8], [15].

In our design, the FOC is emulated by $5^{\text {th }}$-order $\mathrm{RC}$ structure called Foster I, which is demonstrated in Fig. 3. The values of the passive parts are calculated by Oustaloup's algorithm in MATLAB based on selected parameter of $\alpha$, number of the sections of the RC structure, value of the equivalent capacitor and frequency band of approximation validity. The obtained values of the passive parts are summarized in Tab. I. Magnitude and phase responses of the FOC for three values of $\alpha=\beta$ at central frequency $10 \mathrm{kHz}$ are depicted in Fig. 4.

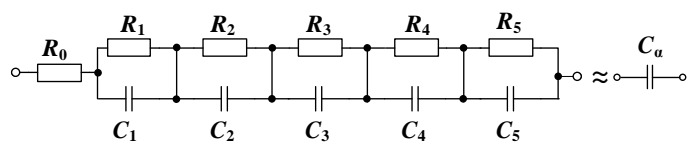

Fig. 3. The $5^{\text {th }}$-order structure approximating the FOC (Fractional-Order Capacitor).

TABLE I

USED VALUES OF RESISTORS AND CAPACITORS IN THE RC STRUCTURE FOR THREE CONSIDERED VALUES OF $\alpha=\beta$.

\begin{tabular}{|c|c|c|c|}
\hline$\alpha[-]$ & 0.3 & 0.5 & 0.8 \\
\hline$C_{\text {eq }}[\mathrm{nF}]$ & \multicolumn{3}{|c|}{6.8} \\
\hline$C_{\alpha}=C_{\beta}\left[\mu \mathrm{F} / \mathrm{sec}^{1-\alpha}\right]$ & 15.5 & 1.7 & 0.062 \\
\hline \hline$R_{0}[\Omega]$ & 588 & 233.9 & 58.8 \\
\hline$R_{1}[\Omega]$ & 365.2 & 241.5 & 65.14 \\
\hline$R_{2}[\Omega]$ & 685.7 & 675.9 & 326.4 \\
\hline$R_{3}[\Omega]$ & 1208.2 & 1734.7 & 1470 \\
\hline$R_{4}[\Omega]$ & 2143.9 & 4545 & 7060 \\
\hline$R_{5}[\Omega]$ & 4328.1 & 15973 & 84232 \\
\hline$C_{1}[\mathrm{nF}]$ & 1.44 & 2.62 & 12.8 \\
\hline$C_{2}[\mathrm{nF}]$ & 4.85 & 5.91 & 16.2 \\
\hline$C_{3}[\mathrm{nF}]$ & 17.37 & 14.5 & 22.6 \\
\hline$C_{4}[\mathrm{nF}]$ & 61.75 & 35 & 29.7 \\
\hline$C_{5}[\mathrm{nF}]$ & 193 & 62.9 & 15.7 \\
\hline & & \multicolumn{1}{|c}{}
\end{tabular}



Fig. 4. Magnitude and phase responses of the FOC implemented by $5^{\text {th }}$-order RC structures for three values of $\alpha=\beta$ at central frequency $10 \mathrm{kHz}$.

The proposed circuit was tested for three values of $\alpha=\beta$ when the FO was set to $10 \mathrm{kHz}$. Using (7), (8), the calculated values of the $g_{\mathrm{m} 1}$ and $g_{\mathrm{m} 2}$ are summarized in Tab. II, which assume ideal active elements. For simulations were also used the following values of auxiliary passive parts: $R_{\mathrm{G}}=R_{1 \mathrm{AUX}}=R_{\mathrm{m}}=1 \mathrm{k} \Omega, R_{2 \mathrm{AUX}}=1 / g_{\mathrm{m} 1}=\{1.315$, $1.656,3.787\} \mathrm{k} \Omega$. The obtained waveforms of the proposed oscillator with non-ideal models of the used active elements for different values of the $\alpha=\beta$ are depicted in Fig. 5. To start up the oscillations, the values of $g_{\mathrm{m} 1}$ had to be modified (Tab. II) due to the influence of parasitic properties of the real (non-ideal) models of the active elements. The modified 
values of $g_{\mathrm{m} 1}=1 / R_{2 \mathrm{AUX}}$ for three values of $\alpha=\beta$ are: $0.787,0.635,0.290 \mathrm{mS}$. The impact of non-ideal properties of the active elements is further analysed in section IV.

TABLE II

CALCULATED VALUES OF THE PASSIVE COMPONENTS FOR THREE VALUES OF $\alpha=\beta$ WHEN FO WAS $10 \mathrm{KHz}$

\begin{tabular}{|c|c|c|c|}
\hline$\alpha=\beta[-]$ & 0.3 & 0.5 & 0.8 \\
\hline \hline$C_{\mathrm{eq}}[\mathrm{nF}]$ & \multicolumn{3}{|c|}{6.8} \\
\hline$C_{\alpha}=C_{\beta}\left[\mu \mathrm{F} / \mathrm{sec}^{1-\alpha}\right]$ & 15.5 & 1.7 & 0.062 \\
\hline \hline $\begin{array}{c}g_{\mathrm{m} 1}=1 / R_{\mathrm{gm} 1}[\mathrm{mS}] \\
\left(\text { modified } g_{\mathrm{m} 1}\right)\end{array}$ & 0.76 & 0.6 & 0.26 \\
\hline$g_{\mathrm{m} 2}=1 / R_{\mathrm{gm} 2}[\mathrm{mS}]$ & $0.787)$ & $(0.635)$ & $(0.290)$ \\
\hline
\end{tabular}

The FO of the circuit in case of $\alpha=\beta=0.3$ and $\alpha=\beta=$ 0.5 equals to $10.251 \mathrm{kHz}$. For circuit with $\alpha=\beta=0.8$ is FO $10.183 \mathrm{kHz}$. The obtained phase shifts between output voltages $v_{2}$ and $v_{1}$ for three values of $\alpha=\beta$ are: 26.27, 44.4 and $70.3^{\circ}$. The phase shifts between output voltages $v_{3}$ and $v_{2}$ for three values of $\alpha=\beta$ are: $26.15,45.6$ and $72.7^{\circ}$. Theoretical phase shifts between $v_{2}$ and $v_{1}, v_{3}$ and $v_{2}$ for three values of $\alpha=\beta$ are: 27,45 and $72^{\circ}$. It obvious that obtained values of phase shifts are very close to the theoretical expectation. The differences are caused by parasitic properties of the used active elements and by rounding of values of resistors and transconductances.

The proposed fractional-order oscillator provides ability to control the FO by changing the values of $g_{\mathrm{m} 1}$ and $g_{\mathrm{m} 2}$. The circuit was tested for four values of the FO: 2.5, 5, 10, and $50 \mathrm{kHz}$. The values of calculated values of the $g_{\mathrm{m} 1}$ and $g_{\mathrm{m} 2}$ are summarized in Tab. III. The frequency range of tunability has been chosen based on validity of the FOC approximation as shown in Fig. 4. In order to extend the range of the frequency tunability of the oscillator, another FOC designed with wide range of the approximation must be used. The obtained waveforms of the circuit with non-ideal simulation models of the active elements for FO tuning are depicted in Fig. 6. To start up the oscillations, the values of the $g_{\mathrm{m} 1}$ had to be modified. These values are stated in parentheses in the Tab. III. The values of the FO for selected output voltage $v_{2}$ are as follows: $2.468,5.164,10.251$, and $50.01 \mathrm{kHz}$. From obtained values of FO is obvious that results correspond with theoretical expectations.

TABLE III

CALCULATED VALUES OF THE TRANSCONDUCTANCES FOR FOUR VALUES OF FO WHEN $\alpha=\beta=0.5$

\begin{tabular}{|c|c|c|c|c|}
\hline FO $[\mathrm{kHz}]$ & 2.5 & 5 & 10 & 50 \\
\hline \hline $\begin{array}{c}g_{\mathrm{m} 1}=1 / R_{\mathrm{gm} 1}[\mathrm{mS}] \\
\left.\text { (modified } g_{\mathrm{m} 1}\right)\end{array}$ & 0.302 & 0.427 & 0.6 & 1.351 \\
\hline$g_{\mathrm{m} 2}=1 / R_{\mathrm{gm} 2}[\mathrm{mS}]$ & $0.326)$ & $(0.46)$ & $(0.635)$ & $(1.46)$ \\
\hline
\end{tabular}

The THD is analysed for three values of $\alpha=\beta$ of the oscillator voltage outputs $v_{1}, v_{2}$ and $v_{3}$ when the FO was set to $10 \mathrm{kHz}$. The demonstration of the spectral analysis of the output voltage $v_{2}$ of the proposed circuit is demonstrated in Fig. 7. The THD was calculated for all values of $\alpha=\beta$ and



(a)

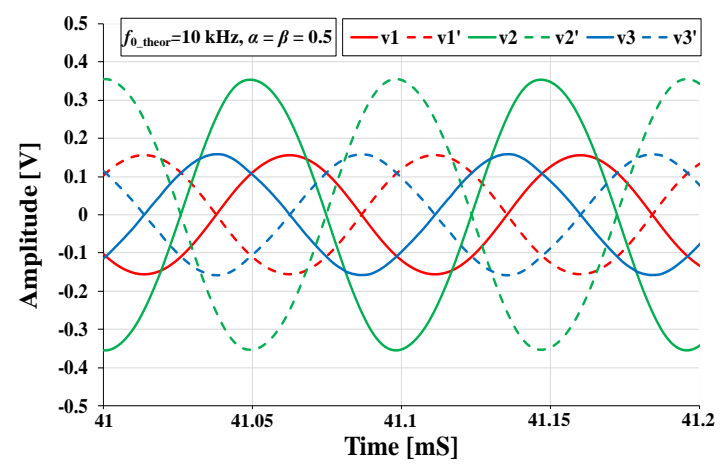

(b)

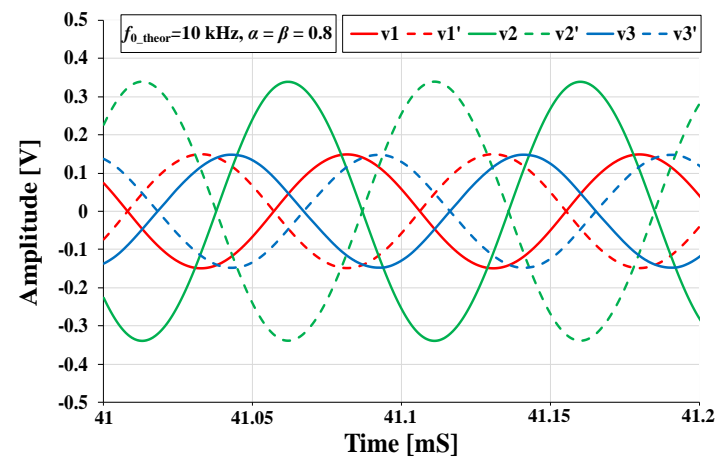

(c)

Fig. 5. Waveforms of the voltage outputs $v_{1}, v_{2}, v_{3}$ and their inverted variants (dashed lines) for three values of $\alpha=\beta$ when the FO (Frequency of Oscilation) was $10 \mathrm{kHz}$ : (a) $\alpha=\beta=0.3$, (b) $\alpha=\beta=0.5$, (c) $\alpha=\beta=0.8$.

output voltages $v_{1}, v_{2}$ and $v_{3}$. The values of THD for $v_{1}$ when $\alpha=\beta=0.3,0.5$, and 0.8 are following: $0.96 \%, 0.71 \%$, and $0.78 \%$. The values of THD calculated for $v_{2}$ are: $1.27 \%$, $1.91 \%$, and $0.5 \%$. The values of THD calculated for $v_{3}$ are: $1.71 \%, 1.93 \%$, and $1.82 \%$. It can be seen that THD is relatively low (below $2 \%$ ) for all values of $\alpha=\beta$ and output voltages.

\section{PARASITIC ANALYSIS}

The parasitic analysis of the fractional-order oscillator was carried out. In Fig 8 are depicted most significant parasitic admittances of the output and input terminals of the used active elements. The input and output parameters of the OTA 


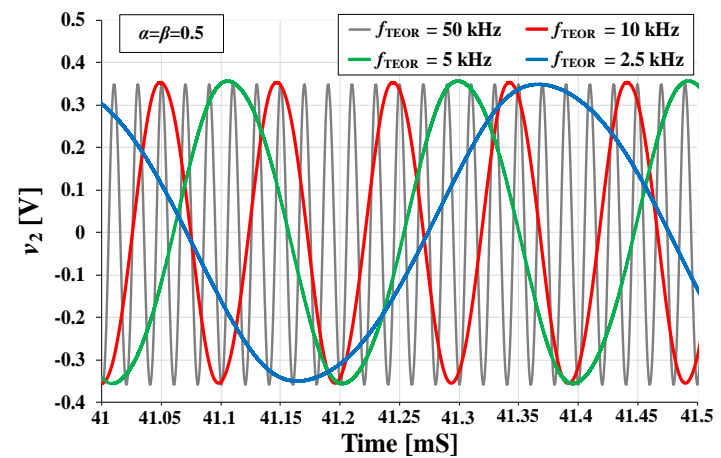

Fig. 6. Waveforms of the voltage output $v_{2}$ for four different values of FO when $\alpha=\beta=0.5$

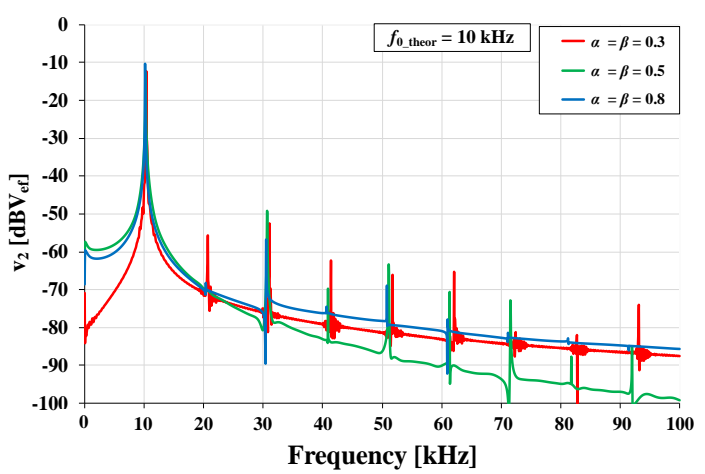

Fig. 7. Spectral analysis of the results of selected voltage $v_{2}$ for three different values of $\alpha=\beta$ when the FO was $10 \mathrm{kHz}$.

element, which is implemented by UCC as is described in section II, are following: $R_{\mathrm{IN}} \approx 1 \mathrm{G} \Omega, C_{\mathrm{IN}} \approx 1 \mathrm{pF}$, $R_{\mathrm{OUT}} \approx 200 \mathrm{k} \Omega, C_{\mathrm{OUT}} \approx 0.6 \mathrm{pF}[13]$. The input and output parameters of the OTRA are: $R_{\mathrm{IN}} \approx 1 \Omega, R_{\mathrm{OUT}} \approx 1.1 \Omega$ [?].

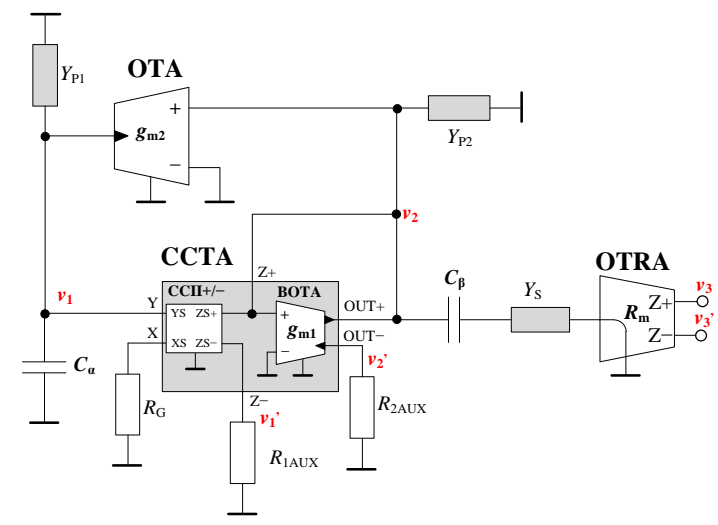

Fig. 8. Scheme of the proposed oscillator from Fig. 1 with parasitic admittances.

The parasitic admittances of the used active elements of the oscillator are given by: $Y_{\mathrm{P} 1}=G_{\mathrm{P} 1}+s C_{\mathrm{P} 1}=1 / R_{\mathrm{OUT}-\mathrm{OTA}}+$ $1 / R_{\mathrm{IN}-\mathrm{CCII}}+s C_{\mathrm{OUT}-\mathrm{OTA}}+s C_{\mathrm{IN}-\mathrm{CCII}}, Y_{\mathrm{P} 2}=G_{\mathrm{P} 2}+$ $s C_{\mathrm{P} 2}=1 / R_{\mathrm{OUT}-\mathrm{BOTA}}+1 / R_{\mathrm{IN}-\mathrm{OTA}}+1 / R_{\mathrm{OUT}-\mathrm{CCII}}+$
$1 / R_{\mathrm{IN}-\mathrm{BOTA}}+\boldsymbol{s} C_{\mathrm{OUT}-\mathrm{BOTA}}+\boldsymbol{s} \boldsymbol{C}_{\mathrm{IN}-\mathrm{OTA}}+\boldsymbol{s} C_{\mathrm{OUT}-\mathrm{CCII}}+$ $s C_{\mathrm{IN}-\mathrm{BOTA}}, Y_{\mathrm{IN}-\mathrm{OTRA}}=G_{\mathrm{P} 3}=1 / R_{\mathrm{IN}-\mathrm{OTRA}}$.

From before mentioned parasitic admittances, $Y_{\mathrm{P} 2}$ has most significant effect on circuit function in comparison with other parasitic admittances. The $Y_{\mathrm{P} 2}$ influences all output voltages of the oscillator. The $G_{\mathrm{P} 2}$ has biggest impact on fulfilment of the condition of oscillation. The parasitic capacitance $C_{\mathrm{P} 2}$ has most significant influence on frequency of the oscillations. The impact of parasitic resistance $R_{\mathrm{P} 2}$ on oscillator function is depicted in Fig. 9. The analysis is performed for output voltage $v_{2}$, three values of the $\alpha=\beta$ and six selected values of the $R_{\mathrm{P} 2}(25,50,100,250,500$, and $5000 \mathrm{k} \Omega)$ when $C_{\mathrm{P} 2}$ $=3.2 \mathrm{pF}$ and FO was $10 \mathrm{kHz}$. From obtained results of the circuit with $\alpha=\beta=0.3$ for six selected values of $R_{\mathrm{P} 2}$, it is obvious that condition of oscillation is fulfilled when $R_{\mathrm{P} 2} \approx 5000 \mathrm{k} \Omega$. In case of oscillator with $\alpha=\beta=0.5$ and $\alpha=\beta=0.8$, the condition of oscillation is not fulfilled for $R_{\mathrm{P} 2}<250 \mathrm{k} \Omega$. From obtained analysis is obvious that the parameter $g_{\mathrm{m} 1}$ or $g_{\mathrm{m} 2}$ must be modified for start up the oscillations because the calculated value of the $R_{\mathrm{P} 2}$, based on parallel connection of the input and output parasitic parameters of the active elements, equals to $83 \mathrm{k} \Omega$.

The impact of $C_{\mathrm{P} 2}$ on FO for three values of the $\alpha=\beta$ is shown in Fig. 10. The analysis was carried out for $C_{\mathrm{P} 2}$ in range from $0.1 \mathrm{pF}$ to $100 \mathrm{pF}$ when $R_{\mathrm{P} 2}$ was $5000 \mathrm{k} \Omega$. It is obvious that higher values of the $C_{\mathrm{P} 2}$ cause frequency shift. Most significant frequency shift is for oscillator with $\alpha=$ $\beta=0.3$. As mentioned above, in the simulation models, the parasitic capacitance $C_{\mathrm{P} 2}$ result from the interconnection of active elements is approximately $3.2 \mathrm{pF}$ only. This causes the error in oscillation frequency $0.58,0.39$ and $0.17 \%(\alpha=\beta=$ $0.3,0.5,0.8)$ only. The results in Fig. 10 show that for higher values of $C_{\mathrm{P} 2}$ the oscillation remains.

\section{CONCLUSION}

The multiphase fractional-order oscillator with controllable FO was designed and analysed in this paper. In case of $\alpha \neq \beta$, the proposed circuit provides three voltage outputs and their inverted variants with phase shift equal to multiple of $\alpha \pi / 2$ and $\beta \pi / 2$. Note that this oscillator with arbitrary phase shift can find the application in signal modulators for example. The fractional-order capacitors, designed by Foster I type of the RC structure, were used instead of standard capacitors in the circuit structure. The proposed oscillator was tested for three values of the $\alpha=\beta$. The circuit provides FO tuning by changing values of $g_{\mathrm{m} 1}$ and $g_{\mathrm{m} 2}$ and was tested for four selected values of FO in range from 2.5 to $50 \mathrm{kHz}$ when $\alpha=\beta=0.5$. The THD of the circuit, which was analysed for three values of the order, is below $2 \%$ for all voltage outputs. The parasitic analysis was also carried out for most significant parasitic admittances of the oscillator. From this analysis was found that the $Y_{\mathrm{P} 2}$ has greatest impact to the circuit function. The parasitic resistance $R_{\mathrm{P} 2}$ influences the condition of oscillation. The parasitic capacitance $C_{\mathrm{P} 2}$ causes the most significant frequency shift compared with other parasitics. The 


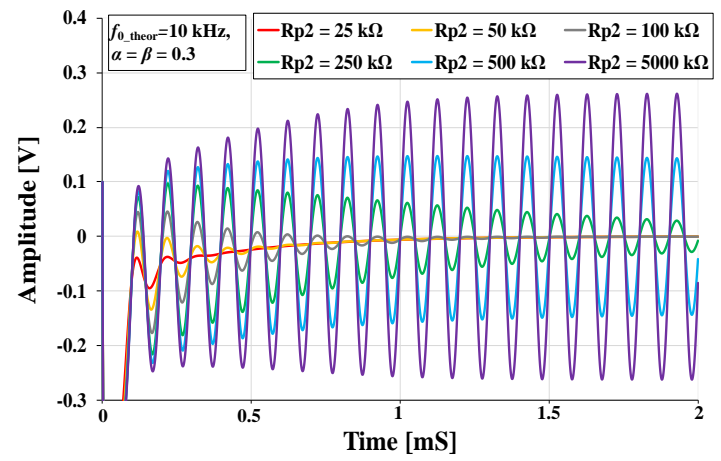

(a)

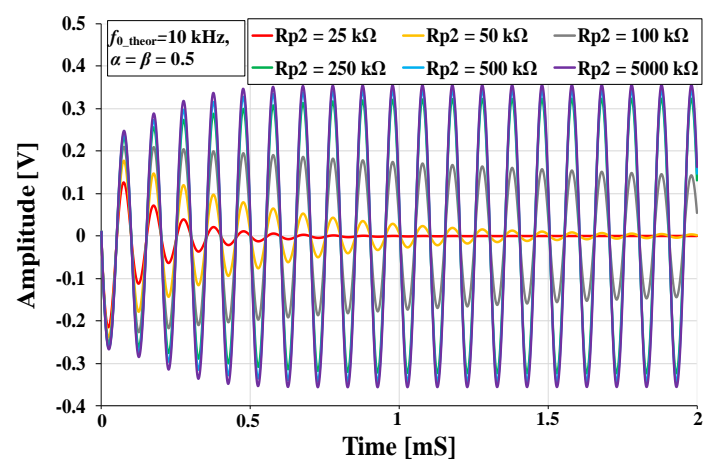

(b)



(c)

Fig. 9. Influence of $R_{\mathrm{p} 2}$ parasitic on waveform of the output voltage $v_{2}$ when $C_{\mathrm{p} 2}=3.2 \mathrm{pF}$ : (a) $\alpha=\beta=0.3$, (b) $\alpha=\beta=0.5$, (c) $\alpha=\beta=0.8$.

function of the circuit is supported by PSpice simulations with non-ideal models of the active elements.

\section{REFERENCES}

[1] A. Elwakil, "Fractional-Order Circuits and Systems: An Emerging Interdisciplinary Research Area", IEEE Circuits and Systems Magazine, vol. 10, no. 4, pp. 40-50, 2010.

[2] J. Munoz-Pacheco, E. Zambrano-Serrano, C. Volos, S. Jafari, J. Kengne, and K. Rajagopal, "A New Fractional-Order Chaotic System with Different Families of Hidden and Self-Excited Attractors", Entropy, vol. 20, no. 8, 2018.

[3] C. Ionescu, A. Lopes, D. Copot, J. A. T. Machado, and J. H. T. Bates, "The role of fractional calculus in modeling biological phenomena: A review", Communications in Nonlinear Science and Numerical Simulation, vol. 51, pp. 141-159, 2017.



Fig. 10. Influence of $C_{\mathrm{p} 2}$ parasitic on frequency of the output voltage $v_{2}$ when $R_{\mathrm{p} 2}=5000 \mathrm{k} \Omega$.

[4] S. Kapoulea, A. M. AbdelAty, A. S. Elwakil, C. Psychalinos, and A. G. Radwan, "Cole-Cole Bio-Impedance Parameters Extraction From a Single Time-Domain Measurement", 2019 8th International Conference on Modern Circuits and Systems Technologies (MOCAST), pp. 1-4, 2019.

[5] S. Khubalkar, A. Junghare, M. Aware, and S. Das, "Unique fractional calculus engineering laboratory for learning and research", International Journal of Electrical Engineering Education, Sep. 2018.

[6] P. Bertsias, C. Psychalinos, A. S. Elwakil, and B. J. Maundy, "Simple Multi-Function Fractional-Order Filter Designs", in 2019 8th International Conference on Modern Circuits and Systems Technologies (MOCAST), 2019, pp. 1-4.

[7] D. Kubanek, F. Khateb, G. Tsirimokou, and C. Psychalinos, "Practical Design and Evaluation of Fractional-Order Oscillator Using Differential Voltage Current Conveyors", Circuits, Systems, and Signal Processing, vol. 35, no. 6, pp. 2003-2016, 2016.

[8] A. Kartci, N. Herencsar, L. Brancik, and K. N. Salama, "CMOS-RC Colpitts Oscillator Design Using Floating Fractional-Order Inductance Simulator", in 2018 IEEE 61st International Midwest Symposium on Circuits and Systems (MWSCAS), 2018, pp. 905-908.

[9] R. Sotner, J. Jerabek, O. Domansky, N. Herencsar, A. Kartci, and J. Dvorak, "Practical Design of Fractional-Order Oscillator Employing Simple Resonator and Negative Resistor", 2018 10th International Congress on Ultra Modern Telecommunications and Control Systems and Workshops (ICUMT), pp. 1-4, 2018.

[10] A. S. Elwakil, A. Agambayev, A. Allagui, and K. N. Salama, "Experimental demonstration of fractional-order oscillators of orders 2.6 and 2.7", vol. 96, pp. 160-164, 2017.

[11] A. Agambayev, A. Kartci, A. H. Hassan, N. Herencsar, H. Bagci, and K. N. Salama, "Fractional-Order Hartley Oscillator", 2018 14th Conference on Ph.D. Research in Microelectronics and Electronics (PRIME), pp. 45-48, 2018.

[12] R. Prokop and V. Musil, "CCTA: a new modern circuit block and its internal realization”, in Proc. of Electronic Devices and Systems IMAPS CS International Conference 2005, pp. 89-93, 2005.

[13] J. Jerabek, R. Sotner, and K. Vrba, "Tunable Universal Filter with Current Follower and Transconductance Amplifiers and Study of Parasitic Influences", Journal of Electrical Engineering, vol. 62, no. 6, Jan. 2011.

[14] D. Biolek, R. Senani, V. Biolkova, and Z. Kolka, "Active Elements for Analog Signal Processing: Classification, Review, and New Proposals", Radioengineering, vol. 17, no. 4, pp. 15-32, 2008.

[15] G. Tsirimokou, "A systematic procedure for deriving RC networks of fractional-order elements emulators using MATLAB", AEU - International Journal of Electronics and Communications, vol. 78, pp. 7-14, 2017.

[16] N. Herencsar, and K. Vrba, "Current conveyors-based circuits using novel transformation method", IEICE Electronics Express, vol. 4, no. 21, pp. 650-656, 2007. 